\title{
THE ROLE OF HR PROFESSIONAL IN TECHNOLOGICALLY ADVANCED HR FUNCTION
}

\author{
NABILA KHAN
}

UGC-JRF Qualified, HR Manager, L\&T Finance, PGDM in HR from LIBA, Chennai, Tamil Nadu, India

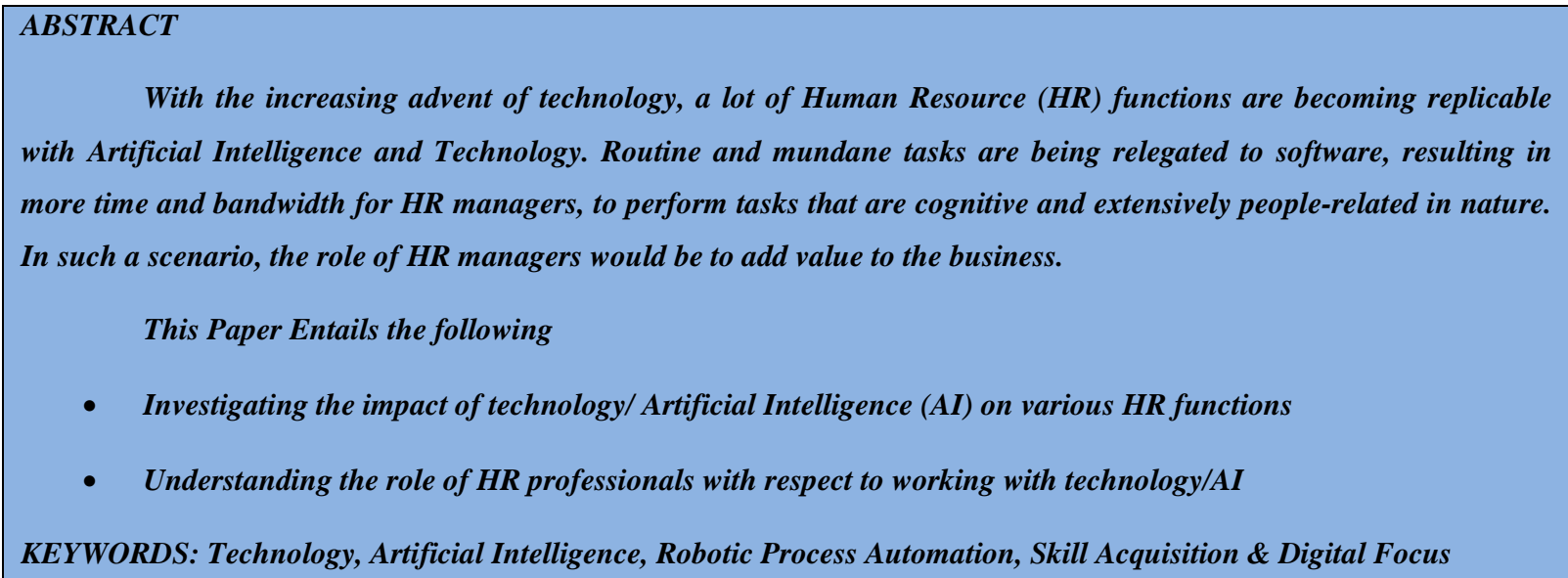

With the increasing advent of technology, a lot of Human Resource (HR) functions are becoming replicable with Artificial Intelligence and Technology. Routine and mundane tasks are being relegated to software, resulting in more time and bandwidth for HR managers, to perform tasks that are cognitive and extensively people-related in nature. In such a scenario, the role of HR managers would be to add value to the business.

This Paper Entails the following

- Investigating the impact of technology/ Artificial Intelligence (AI) on various HR functions

- Understanding the role of HR professionals with respect to working with technology/AI

KEYWORDS: Technology, Artificial Intelligence, Robotic Process Automation, Skill Acquisition \& Digital Focus

Received: Aug 07, 2017; Accepted: Aug 21, 2017; Published: Aug 30, 2017; Paper Id.: IJHRMROCT20171

\section{INTRODUCTION}

For the past few years, there have been ongoing discussions on technology replacing humans in the people function of HR. Predictive Behaviour Technology, Talent Analytics and algorithms have a significant impact on HR. Yet, there is an issue, as technology is not completely reliable.

Today's HR professional, resides somewhere between the cold precision of data and the warmth of personal association. For years, we've observed HR transform in front of our eyes: technology has seeped into our exchanges in the workplace; from recruiting and training, to performance management, resulting in many speculating, how - and if - HR professionals still have a place in contemporary organisations.

According to the highlights of Deloitte's Global Shared Services Survey (2017)1, "Robotic process automation is a rapidly emerging technology, that will fundamentally change how shared services operate, slashing the effort for routine tasks and enabling advanced cognitive applications, that augment or replace human judgment in knowledge-based processes".

Organisations will continue to require lesser HR professionals, to perform transactional administrative tasks. Emerging information and communication technologies will continue to be used, to increase the effectiveness of HR administrative jobs such as, employee record-keeping, payroll, entry-level staffing, and benefits administration. As HR professionals become increasingly competent to contribute to business success, they will grow to be more balanced in their approach towards work.

Technology can make an employee's job better, by analysing what works and what doesn't. It can be used to free up the HR professional's time, to focus on strategy and developing new policies. In fact, it can even give 
recruiters tools to make the process more human, because effective HR requires a human touch. This is the part of the balance between Science and Art. Taking into account, the data as a paint-by-numbers project: analytics gives you the colours and numbers—-but you're still the one who has to paint the picture.

\section{TECHNOLOGY IN VARIOUS FUNCTIONS OF HR}

\section{Recruitment}

We have reached a day and age where technology is aiding recruitment efforts, by saving time and resources. Among the many advantages of going digital for hiring are improved productivity, less risk of a bad candidate match and an increase in strategic hiring decisions. The methodologies that are currently in use for recruitment and selection are:

- Social Media is an efficient means that can be used, to connect with prospective hires. Using social media, openings can be advertised, new hires introduced, and candidates engaged. A mobile-compatible medium makes recruiting effortless.

- Applicant Tracking Systems (ATS), aids in sorting and managing contenders for a particular position. ATS can be used, for posting job vacancies, scrutinising resumes, and keeping a track of where the aspirants are in the hiring process. It can be used for importing resumes right into the software, for organising candidates according to their level of competency.

- Digital Templates can be used to provide a consistent and seamless look, to the hiring efforts of the business and increase the pace of document creation. Spending manual effort into creating and sending every offer letter and rejection letter is tedious, as well as time- consuming. Communicating with candidates can become more efficient with time and money saved, using such templates.

Google Hire is a type of recruitment software that would aid organisations not only in performing most functionalities of an ATS, but also in other tasks like candidate nurturing \& discovery. It includes effortless features like every interviewer being able to access the other interviewer's comments, only after they have given their own feedback. This feature has been built in, to avoid bias and group think. Another example of the features included in Google Hire is, when you type "Sales Manager", the search engine inside this software will discern what you mean, ensuring its understanding of the way the search is done and what is required by people at the time they are looking for something. The recruitment market is segmented into candidates, with varying experience who are from diverse industries. In order to work with such varying requirements and to harness the best of the technology, Microsoft acquired Linked in, Aon Hewitt acquired Cocubes and Sales force is moving towards HR technology. These trends show increased integration of technology and traditional HR management.

\section{Performance Management}

Performance Management, in the form of a yearly appraisal has of late lost its lustre to more effective practices. Leaders and employees across industries view performance management as lengthy, subjective, intimidating and unhelpful, for the development of the employees. In such scenarios, it doesn't do much to develop the employees. It may even pull down their performance, as they struggle with scores and ratings, fret about compensation, and attempt to comprehend feedback. Collecting substantial amount of data is essential for the new processes; this is primarily because, many employees are of the opinion that, the present evaluation practices are fraught with subjectivity. Organisations can get 
improved information, by making use of systems that crowd-source and gather details on the performance of employees and their teams, instead of depending on an annual, vague analysis of individuals. Enhanced insights on performance are obtained, by collecting performance data throughout the year.

For example, Zalando, a top European e-retailer, is at present employing a tool that crowd-sources structured and unstructured performance feedback simultaneously from conferences, problem-solving meetings, concluded assignments, launches, and campaigns. Staff members can request for opinion and feedback from superiors, peers, and internal "customers", with the aid of an online app that allows individuals to provide both constructive and critical comments in a good-humoured and engaging way. Depending on the exposure of the feedback contributor to the requestor, the system assigns weights to the responses received. The tool is easy to use, with a list of feedback questions that can be answered instinctively, by moving a slider displayed on the mobile device. As the information is assimilated in real time, it tends to be more precise, as compared to annual reviews, where peers and superiors must strain themselves, in order to recall particulars about the individuals they assess.

Employees at General Electric (GE), now use a tool, called PD@GE, which is similar and assists supervisors in keeping track of the organisation's goals, even as they alter year-round. It enables requests for feedback and stores details of when it is received. The employees receive qualitative as well as quantitative details about their performance, which helps them realign swiftly, throughout the year. This tool, however, does not substitute for conversations on performance between supervisors and employees. As an alternative, these conversations revolve around the feedback of colleagues, supervisors, and the staff themselves, about what helped and what did not help in achieving goals. As organisations mechanise activities and insert AI and machine learning to the mix, the value of this data will improve rapidly, and it will be collected much more efficiently. These performance management tools can also categorise the top performers, more accurately.

Towards the year end, Zalando's tool will recommend the top 10 percent performing employees, by examining the accumulated feedback details. Supervisors have the option of altering the size of the pool of the top performers. This tool will provide the management with factual and objective information, to discern the truly distinguished employees. It can also be used to identify those employees who are lagging behind and provide help in their development.

\section{Learning and Development}

With the rapidly evolving technology, there is a pressing need for employees to keep up by upgrading their skills. Owing to the paucity of time and resources to achieve skill enhancement, learning and development teams are required to come up with pioneering techniques, to train employees promptly and with minimum resources.

Organisations like Piramal, GE, Genpact, Ericsson and many others have moved on from standalone traditional programs to using a blended form of learning, with the aid of technology. Learning techniques now include gamification, experiential learning environments and the "flipped classroom" (theory to be studied at home/out of the classroom via videos; enrichment and reinforcement activities to be conducted inside the classroom). These techniques have proved to be immensely beneficial in leadership development. In this age of digitisation, not only is the learning function responsible for creating the right content, but also for compiling the right content, that is sufficient, timely and apt for the employee who is learning.

Peer-to-peer learning is also encouraged for sharing ideas, knowledge and expertise, via a knowledge sharing 
portal. Apart from this, organisations are also partnering with Massive Open Online Courses (MOOCs), learning material suppliers and organisations that conduct research in order to leverage advances in technology, for learning and development. This enables efficient utilisation of manpower in the Learning and Development team and enhances their productivity.

\section{Employee Engagement}

Employee engagement signifies the commitment of an employee, towards his/her organisation and the amount of discretionary effort he/she is willing to put in to achieve the goals of the organisation. The annual engagement survey is no longer the only means by which, an organisation gets to know its employees' opinions. Over the years, the annual ritual of a survey has turned into a mere formality, with many man-hours spent in designing and administering the survey, which ultimately yields dated results. The advent of technology has paved the way, for real-time surveys. Contemporary survey platforms let organizations put together and deploy surveys, within a few minutes. Such fast means of collecting real-time data, enables the HR team to record the opinions of employees as and when needed - be it post a change in management, an announcement of a new strategy, or even an external event. A plethora of these survey platforms are equipped with advanced reporting and analytics functions, which enable the organisation in deciding the course of action within a short span of time.

The latest offering in the employee engagement segment is that of employee voice apps. "Always on" apps are swiftly achieving popularity as a medium that enables the capturing of employee opinions. A common widespread attribute with many of these apps is the facility for employees, to directly communicate with the leaders of the organisation. The apps help to meet the needs and requirements of HR and business leaders, at various levels with customised dashboards and analytics. For example, there would be a separate dedicated dashboard each, for the CEO, the business leaders, and the HR Managers of the company.

There are various ways in which employee engagement data is collected using technology. Organisations are finding intelligent means of mining and analysing the voice of the employees, from these data sources. Sentiment analysis algorithms, analysis of social media activity and word cloud, along with HR system analytics are a few of the widely used methods, for analysing this kind of data. Certain platforms use various enterprise systems and the public domain, to provide fascinating engagement results in the form of the general mood of the employees, organisation-level issues, along with policies, word clouds and popularity rankings of the leaders. These methodologies will lead to an increase in the level of engagement and will help nurture an environment where employees do not feel isolated.

\section{Embedding Diversity using Technology}

In the past few years, "diversity" has been the in-thing, in the corporate world. An endeavour by organisations to look progressive, but rarely done with the spirit intended. Nevertheless, it has transformed into a strategic priority, intended to make organisations more competitive. According to a research by McKinsey (2015) $)^{13}$, companies are 35 percent more likely to have financial returns, above their respective national industry medians, when they are in the top quartile for ethnic and racial diversity. They are also 15 percent more likely to have financial returns, above their respective national industry medians, when they are in the top quartile for gender diversity.

Adoption of innovative technology by an organisation helps it get better insights, with respect to varied occurrences in different phases of recruitment; the types of people the market attracts for different types of roles, the way 
diverse candidates are reviewed by varying departments and managers and the way these aspirants advance in the course of the recruitment process, down to the last level of detail. Such technology can examine the rates of job offers, by particular interviewers, which in turn helps to unveil unconscious and conscious bias.

A lot of enterprising organisations have turned online, for their recruitment process in order to provide for a bigger prospective workforce that may be unable to attend an interview, due to disabilities or location constraints. Only bringing on board diversity is not sufficient, technology should enable monitoring and gauging the effect of hiring diversity and strive for constant advancement; companies are putting in millions, to keep tabs on the performance of their diversity initiatives. Organisations can conduct an analysis of the existing internal data and surveys to i) Comprehend how employees of different demographics make progress, ii) Fill any gaps in terms of supporting employee progress, iii) Identify the faction of people that has successfully progressed on its career path and iv) Positively impact the growth of other groups in the organisation.

The latest technology to include diversity is "Rare Recruitment's Contextual Recruitment System". It is intended to encourage social mobility that will enable organisations to take personal circumstances, as well as the economic background of an aspirant into consideration. This would enable an organisation, to identify distinguished applicants regardless of their circumstances. Technology is progressively helping eliminate residual obstacles. Among the most striking technological developments to help overcome discrimination is, technology that makes intelligent conversation to help people, not familiar with English feel at ease with the language. This will eventually help organisations, move towards a truly global workforce.

\section{CONCLUSIONS}

According to Erica Volini, Pascal Occean, Michael Stephan and Brett Walsh in their report titled "2017 Deloitte Global Human Capital Trends: Rewriting the rules for the digital age" 15 , "HR is leading digital transformation and the shift is happening rapidly, as HR leaders are being pushed to take on a larger role in helping to drive the organization to "be digital," not just "do digital."

- 56 percent of companies surveyed are redesigning their HR programs to leverage digital and mobile tools

- 51 percent of companies are currently in the process of redesigning their organizations for digital business models

- 33 percent of surveyed HR teams are using some form of AI technology to deliver HR solutions

- 41 percent are actively building mobile apps to deliver HR services"

With organisations trying to be more proficient and productive, the pursuit of strategies and competencies to implement these strategies, are of critical importance in generating sustainable competitive advantages. As Mike Ulrich, David Kryscynski, Dave Ulrich, Wayne Brock bank and Jacqueline Slade have talked about in their "2016 HR Competency Mode"16, strategic contribution, personal credibility, HR delivery, business knowledge and HR technology, are all pivotal to HR professionals becoming effective business partners and players.

To build a digital mindset, technical expertise and digital perspicacity are the two skills needed to ensure that, an organisation embraces the future. In order to create these capabilities, HR primarily needs to have an outside-in approach. Till date, the HR function has been focused internally i.e., on evolving the capability of employees, as per current requirements. Conversely, the present scenario demands an understanding of ever-altering business requirements and the 
recommendation of timely solutions that are effective and budget-friendly.

As per the report, "2017 Deloitte Global Human Capital Trends: Rewriting the rules for the digital age" 15 , "While none of the HR's prior responsibilities have gone away, HR departments today are under pressure to rewrite the rules by redesigning talent practices, from recruiting to leadership to performance management; by experimenting with digital apps; and by building a compelling employee experience."

The recent digital focus for HR is not merely technology; but also the way we serve and support employees. The world, our employees live in is digital and if we do not become a part of it, then we cannot really do our jobs. For example: we, in HR, need to learn how to develop mobile apps; we can make use of design thinking in our jobs; we should think about "experiences" and not "programs"; and, we need to learn how to leverage data, in a more strategic way. People do not want to look at dashboards; they want their computers to "tell them" or "recommend" what to do- based on data. In the present business environment, where routine tasks are being assigned to technology and the cerebral work is the people's responsibility, the HR function will become more strategic than ever before. This will mean, developing digital capabilities in which an organisation's activities, people, culture, and structure are in sync and aligned towards its set of goals.

\section{REFERENCES}

1. Deloitte, Global Shared Services, 2017 Survey Report. https://www2.deloitte.com/us/en/pages/operations/articles/sharedservices-survey.html

2. Sunil J. Ramlall, University of St. Thomas (2006). Identifying and Understanding HR Competencies and their Relationship to Organizational Practices, Applied H.R.M. Research, 2006, Volume 11, Number 1, pages 27-38, http://www.xavier.edu/appliedhrmresearch/2006-Summer/11_1_\%20Ramlall\%20(pages\%2027-38).pdf

3. Ulrich, D., \& Dulebohn, J. H. (2015). Are we there yet? What's next for HR? Human Resource Management Review, 25(2), 188-204. DOI: 10.1016/j.hrmr.2015.01.004//

4. Josh Bersin (2015),Bersin by Deloitte, Predictions for 2016,A Bold New World of Talent, Learning, Leadership, and HR Technology Ahead

5. Meghan M Biro (2016).Will technology replace HR in 2016, http://switchandshift.com/will-technology-replace-hr-in-2016//

6. Michael Gretczko and Rajesh Attra (2016).Can robots replace HR?, https://hrtimesblog.com/2016/11/18/can-robots-replace$\underline{h r l}$

7. How Technology in the Recruitment and Selection Process Can Save You Money http://blog.rpoassociation.org/blog/how-technology-in-the-recruitment-and-selection-process-can-save-you-money

8. Amol Pawar (2017).Google enters HR Tech with Google Hire https://www.peoplematters.in/article/recruitment/google-entershr-tech-with-google-hire-15914

9. Amol Pawar (2017).Decoding the future of job boards,https://www.peoplematters.in/blog/recruitment-processoutsourcing/decoding-the-future-of-job-boards-15035

10. Boris Ewenstein, Bryan Hancock, and Asmus Komm, McKinsey (2016). Ahead of the curve: The future of performance management, $\quad$ http://www.mckinsey.com/business-functions/organization/our-insights/ahead-of-the-curve-the-future-ofperformance-management

11. People Matters \& Simplilearn Study (2017). Skill acquisition for the digital age, https://www.peoplematters.in/download/hr- 
technology/skill-acquisition-for-digital-age-people-matters-simplilearn-study-16014

12. Philip Kushmaro (2016). The top 5 apps to drive employee engagement, http://www.huffingtonpost.com/entry/the-top-5-appsto-drive-employee engagement us 5774bfd4e4b0ee1c313dc486

13. Vivian Hunt, Dennis Layton and Sara Prince, McKinsey \& Company (2015). Diversity Matters, http://www.mckinsey.com/business-functions/organization/our-insights/why-diversity-matters

14. Chandan Chataraj (2017). Leveraging technology to transform diversity for performance, https://www.peoplematters.in/blog/performance-management/leveraging-technology-to-transform-diversity-for-performance$\underline{16029}$

15. Erica Volini, Pascal Occean, Michael Stephan, Brett Walsh, Deloitte (2017). 2017 Deloitte Global Human Capital Trends: Rewriting the rules for the digital age, https://dupress.deloitte.com/dup-us-en/focus/human-capital-trends/2017/digitaltransformation-in-hr.html

16. Mike Ulrich, David Kryscynski, Dave Ulrich, Wayne Brockbank and Jacqueline Slade (2016).2016 HR Competency Model, http://www.apg.pt/downloads/file954 pt.pdf

17. Gerald C. Kane, Doug Palmer, Anh Nguyen Phillips, David Kiron, Natasha Buckley, Deloitte (2016). Aligning the organization for its digital future,https://dupress.deloitte.com/dup-us-en/topics/emerging-technologies/mit-smr-deloittedigital-transformation-strategy.html

18. Jenny Roper (2016). HR: Will a robot take HR jobs, http://www.hrmagazine.co.uk/article-details/will-a-robot-take-hrjobs\#comment-22811 
